Check for updates

Cite this: RSC Adv., 2018, 8, 8442

Received 8th December 2017

Accepted 13th February 2018

DOI: 10.1039/c7ra13154b

rsc.li/rsc-advances

\section{Improved performance of optical phased arrays assisted by transparent graphene nanoheaters and air trenches}

\author{
Yubing Wang, (D)* Lei Liang, Yongyi Chen, Peng Jia, Li Qin, Yun Liu, Yongqiang Ning \\ and Lijun Wang
}

In this paper, high-performance optical phased arrays (OPAs) assisted by transparent graphene nanoheaters and air trenches have been designed and simulated. By directly locating graphene nanoheaters on silicon waveguides, heating efficiency is enhanced by $62.96 \%$ compared to conventional structures with $1 \mu \mathrm{m}$ $\mathrm{SiO}_{2}$ overlays, and is further enhanced by a factor of $200 \%$ by the presence of air trenches. Thanks to the high thermal conductivity of graphene, a record-high operation speed on the order of $200 \mathrm{kHz}$ is realized. Power consumption for $\pi$ phase shift is $4.65 \mathrm{~mW}$, approximately half of that of the state-ofthe-art OPAs. By introducing air trenches, thermal crosstalk is significantly reduced, resulting in an enlarged fill factor. In addition, a novel beam steering scheme in the $\theta$ direction is proposed. By applying a $30 \mathrm{~mW}$ heating power, a temperature gradient along antennas is generated and beam steering of $2.3^{\circ}$ is achieved, satisfying applications such as long-range collision avoidance for autonomous driving.

\section{Introduction}

Optical beam shaping and steering is indispensable in many applications such as LIDAR (light detection and ranging) systems for three-dimensional imaging and mapping and freespace optical communication. ${ }^{1}$ Solid-state beam steering is preferable due to its excellent stability, compact size and reduced weight. Recently, T. Cao et al. theoretically simulated arrays of gradient $\mathrm{Au} / \mathrm{Bi}_{2} \mathrm{Se}_{3} / \mathrm{Au}$ nanostructures and other metamaterials and achieved continuous beam steering of $18^{\circ} .^{2-6}$ However, these exciting results require further experimental research before industrial applications. Optical phased arrays (OPAs) based on arrayed waveguides fabricated via CMOS (complementary metal oxide semiconductor) technology have been considered as one of the most promising technologies to realize solid-state LIDAR system due to their acceptable beam steering angle and potentially reduced cost.

OPAs have been intensively studied in numerous publications. $^{7-16}$ Beam steering in $\phi$ direction (i.e. direction perpendicular to waveguide array, as shown in Fig. 1(a)) is achieved by applying a fixed phase shift between adjacent antennas (also referred as emitters) according to the far-field Fraunhofer diffraction, while beam steering in $\theta$ direction (i.e. direction along waveguide array) is accomplished either by wavelength tuning using the dispersive nature of grating or by fabricating two-dimensional (2D) OPAs.

State Key Laboratory of Luminescence and Applications, Changchun Institute of Optics, Fine Mechanics and Physics, Chinese Academy of Sciences, Changchun 130033, P. R. China. E-mail: wangyubing@ciomp.ac.cn
Generally, Si-based OPAs are thermo-optically tuned and the phase shift is determined by

$$
\Delta T \frac{\partial n}{\partial T} \frac{2 \pi}{\lambda} L
$$

where $\Delta T$ is temperature difference, $\frac{\partial n}{\partial T}$ is thermo-optic coefficient of $\mathrm{Si}, L$ is heating length and $\lambda$ is wavelength in vacuum. ${ }^{7}$

Metal heaters are widely used in silicon thermo-optic phase shifter. ${ }^{12,13,16}$ However, due to strong optical absorption, metal heaters must be separated from waveguides by thick $(\sim 1 \mu \mathrm{m})$ overlay such as $\mathrm{SiO}_{2}$, making metal heaters power-inefficient. In addition, poor heat conductivity of $\mathrm{SiO}_{2}$ hinders heat transfer, prolonging heating and cooling time. Recent breakthrough in silicon OPAs utilizes an adiabatic bend to insert $\mathrm{p}-\mathrm{n}$-junction heaters into $\mathrm{Si}$ waveguides., ${ }^{7, \mathbf{8} 10,15}$ However this approach not only requires careful design, cutting-edge fabrication technique but also may result in optical loss due to Si doping and the adiabatic bend.

As mentioned above, 2D beam steering requires either wavelength tunable laser diode or 2D OPAs. First, it is challenging to design and fabricate chip-integrated semiconductor laser diode with high power, narrow linewidth and wide tunable range. Wavelength-tunable lasers based on silicon platform have only been realized as proof-of-concept demonstrations. ${ }^{1}$ Moreover, wavelength tuned OPAs are generally fed by edge coupling, ${ }^{7,9,12,16}$ resulting in large coupling loss ( $>3 \mathrm{~dB})$, due to the relatively small dimensions of $\mathrm{Si}$ waveguides compared to optical fibers. Furthermore, although 2D OPAs can achieve 2D beam steering using single wavelength, the major disadvantages are the dramatically increased on-chip power consumption, design and 


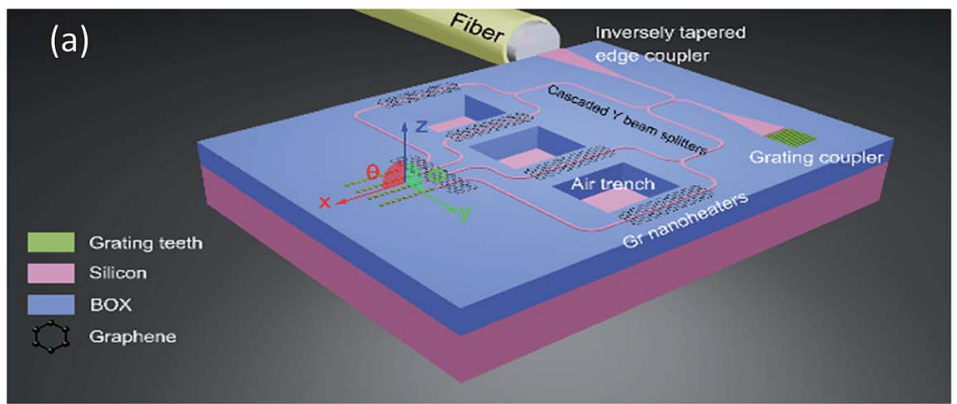

(b)

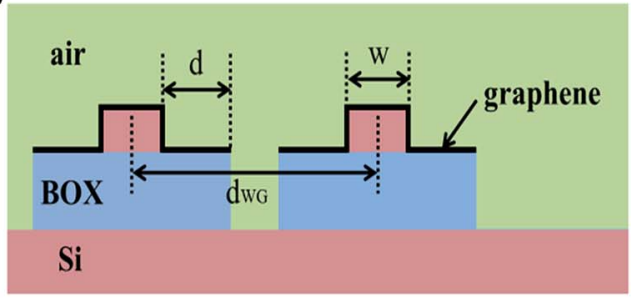

Fig. 1 (a) Schematic of the proposed structure (not to scale). (b) Schematic cross-section of the phase-shifting region.

fabrication complexity, and more sophisticated driving circuits. ${ }^{1,17}$

To conclude, it is imperative to realize thermo-optic OPAs with high power-efficiency, fast operation speed and ease of design and fabrication, and a novel low-power beam steering scheme in the $\theta$ direction without wavelength tunning.

Graphene, a layer of carbon atoms arranged in hexagonal lattice, has received tremendous research interest since its first realization. ${ }^{18-20}$ Experimental results show that graphene has a uniformly high transmission of $97.7 \%$ for wide optical spectrum, from $300 \mathrm{~nm}$ to $2500 \mathrm{~nm} .{ }^{21}$ The transparent nature of graphene allows us directly locating graphene on Si waveguide without any materials in between. Thus heat generated from graphene could transfer directly to $\mathrm{Si}$ waveguide, making graphene nanoheaters more power-efficient. ${ }^{22}$ In addition, the thermal conductivity of graphene is measured to be $5300 \mathrm{~W} \mathrm{~m}^{-1} \mathrm{~K}^{-1},{ }^{23-25}$ over $10^{3}$ times higher than that of silica and air, which are $1.38 \mathrm{~W} \mathrm{~m}^{-1} \mathrm{~K}^{-1}$ and $0.023 \mathrm{~W} \mathrm{~m}^{-1} \mathrm{~K}^{-1}$, respectively. Such high thermal conductivity facilitates heat conduction in the heating and cooling process, making graphene nanoheaters friendlier for high-speed operating situation. Moreover, graphene is compatible with CMOS fabrication process ${ }^{26}$ making graphene nanoheaters readier for large-scale photonic integration circuits (PICs).

In this paper, we demonstrate improved performance of OPAs assisted by graphene nanoheaters. We show that heating efficiency of the graphene contact heating structure is improved by $62.96 \%$ compared to conventional structure with upper cladding. Key parameters including thermal crosstalk, operation speed and power consumption are simulated and the proposed structure is optimized accordingly. In addition, a novel beam steering scheme in the $\theta$ direction is demonstrated.

\section{Simulation and design}

\section{Device structure}

Schematic of the proposed structure is shown in Fig. 1(a). We only draw four channels for clarity. The cross-section of the phase-shifting region, composed of Si rectangular waveguide, air trenches and graphene transparent nanoheater locating directly on the surface of waveguide, is schematically shown in Fig. 1(b). The substrate is an SOI (silicon-on-insulator) substrate with intrinsic $220 \mathrm{~nm}$ top $\mathrm{Si}$ and $2000 \mathrm{~nm}$ buried oxide (BOX). The choice of this standard SOI substrate mainly depends on commercial availability and compatibility with CMOS PICs foundries, imec-ePIXfab for example. Three independent etching steps are required to manufacture the passive elements: a full etch of $220 \mathrm{~nm}$ Si for waveguides, cascaded beam splitters, tapers and air trench windows, a second of $50 \mathrm{~nm}$ Si for the grating couplers and a third of $2000 \mathrm{~nm}$ silica for the air trenches. Large area graphene sheet is transferred onto $\mathrm{Si}$ waveguide by the well-established transfer methods. ${ }^{27-29} \mathrm{Gra}-$ phene nanoheaters are patterned by standard optical lithography followed by oxygen plasma etching. The width of graphene nanoheater is $(2 d+w+440) \mathrm{nm}$. Throughout this paper, area of the patterned graphene nanoheaters is $100 \mu \mathrm{m}^{2}$ and heating power applied to graphene nanoheater is $1 \mathrm{~mW}$, unless otherwise mentioned.

\section{Optical field simulation in $\mathrm{Si}$ waveguide}

Though only single-layer atom thick, graphene has uniformly strong light absorption for near infrared light $(\sim 2.3 \%) .{ }^{21}$ When evanescently coupled with $\mathrm{Si}$ waveguide, the absorption coefficient of graphene could be further enhanced..$^{30}$ Fortunately, thanks to the relatively low density state of graphene, optical absorption could be eliminated by appropriate doping of graphene. According to the Pauli blocking effect, if the doping level of graphene is high enough that the Fermi level of graphene exceeds $\pm \frac{1}{2} h \nu$ regime, where $h \nu$ is energy of incident photons, graphene becomes totally transparent. ${ }^{31-34}$ Moreover, numerous publications have discussed methods of uniform doping of graphene, including gate doping ${ }^{35}$ ion doping ${ }^{36}$ and chemical doping. ${ }^{37,38}$ Therefore, the imaginary part of the refractive index of graphene is assumed zero. In addition, since the thickness of single-layer graphene is $0.34 \mathrm{~nm}$, far smaller than the height of Si waveguide, we could reasonably believe that graphene has negligible influence on optical field distribution. Thus in the optical field simulation, we simplified the model by neglecting the presence of graphene.

The far-field emission pattern of an OPA equals to the farfield pattern of one emitter, or the "envelope", multiplied by an array factor. ${ }^{7,10}$ In order to obtain large field of view (FOV), the envelope should be as wide as possible. Since the width of the envelope is reversely proportional to the width of emitter, 
narrow waveguides are preferable. ${ }^{9}$ Thanks to the large refractive index difference between $\mathrm{Si}$ and $\operatorname{BOX}(\sim 2)$, the propagation loss of optical field is negligible for waveguide widths greater than $500 \mathrm{~nm}$. Therefore, we choose $w=500 \mathrm{~nm}$ for simulation, enabling single-mode condition, low propagation loss and wide envelop.

Neglecting optical absorption of $\mathrm{Si}, \mathrm{BOX}$ and air, the real parts of refractive indexes at $1550 \mathrm{~nm}$ are set at 3.48, 1.45 and 1.00 , respectively. The simulated optical field distribution for transverse-electric (TE) mode is shown in Fig. 2.

\section{Thermal field simulation in phase-shifting region}

In order to clearly demonstrate the merit of graphene nanoheaters which allows for direct contact heating Si waveguide, we first consider the structure without air trenches, which is schematically shown in Fig. 3(a), as a comparison to the conventional structure with $1 \mu \mathrm{m}$-thick $\mathrm{SiO}_{2}$ overlay, as shown in Fig. 3(b).

The thermal field is associated with the heating efficiency of graphene nanoheater. Given a fixed heating power $(1 \mathrm{~mW})$, a higher waveguide core temperature $\left(T_{\text {WGcore }}\right)$ indicates a higher heating efficiency. ${ }^{25}$ Simulations of thermal field are carried out by a commercial software COMSOL which is based on Finite Element Method. The simulated thermal field distribution of the proposed structure with graphene nanoheaters and the conventional structure are simulated, as shown in Fig. 4(a) and (b), respectively. Here, graphene nanoheater are modeled as thin layers and widths of graphene nanoheaters are $1.94 \mu \mathrm{m}(d=500)$ for both simulations. Heat sources are defined by boundary heat sources. The thermal conductivity for air, $\mathrm{Si}, \mathrm{SiO}_{2}$ and graphene are $0.023 \mathrm{~W} \mathrm{~K}^{-1} \mathrm{~m}^{-1}, 130 \mathrm{~W} \mathrm{~K}^{-1} \mathrm{~m}^{-1}$, $1.38 \mathrm{~W} \mathrm{~K}^{-1} \mathrm{~m}^{-1}$ and $5300 \mathrm{~W} \mathrm{~K}^{-1} \mathrm{~m}^{-1}$, respectively, and the ambient temperature is $293.15 \mathrm{~K}$.

As shown in Fig. 4, in the conventional structure, $T_{\text {WGcore }}$ is 298.63 K, corresponding to a temperature elevation of $5.48 \mathrm{~K}$. In the proposed graphene contact-heating structure, $T_{\text {WGcore }}$ is

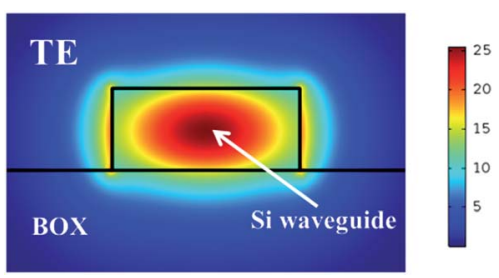

Fig. 2 Optical field distribution for TE mode. The width and height of Si waveguide are $500 \mathrm{~nm}$ and $220 \mathrm{~nm}$, respectively. (a)

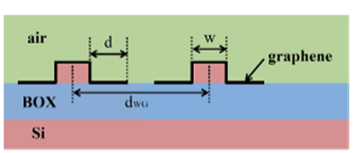

(b)

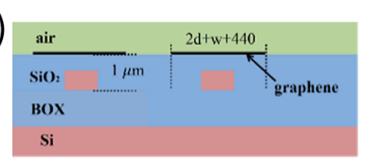

Fig. 3 (a) Schematic of graphene contact-heating structure. (b) Schematic of the conventional structure with $1 \mu$ m-thick top $\mathrm{SiO}_{2}$ cladding layer.
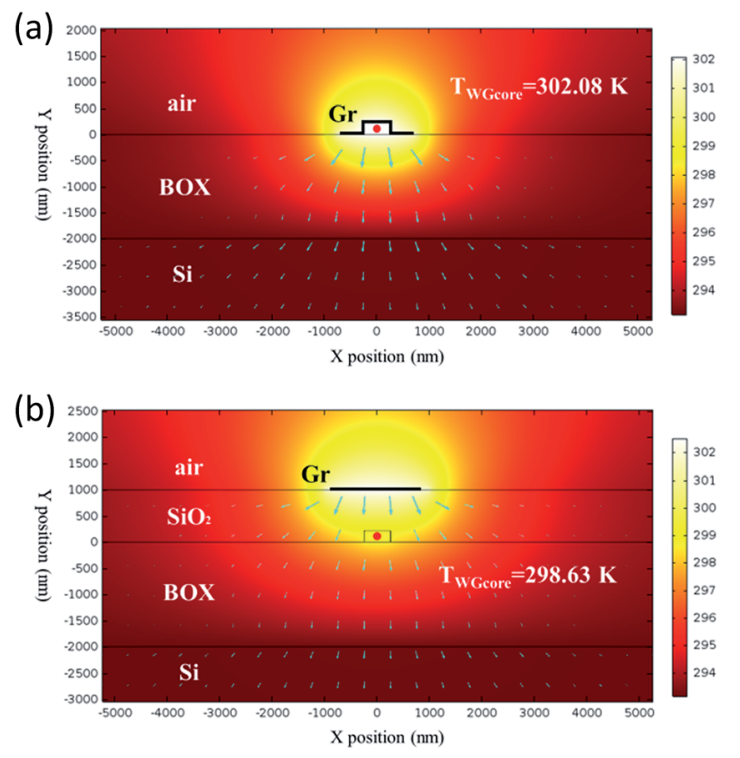

Fig. 4 Simulated thermal field distribution of the proposed structure with graphene contact heating (a) and the conventional structure with $1 \mu \mathrm{m}$-thick top $\mathrm{SiO}_{2}$ cladding layer (b). Blue arrows shows the directions of heat flux.

$302.08 \mathrm{~K}$, corresponding to a temperature elevation of $8.93 \mathrm{~K}$, indicating an enhancement in heating efficiency of $62.96 \%$ is obtained. In the graphene contact-heating configuration, heat flux conducts directly to waveguide without any heat dissipation, giving rise to a higher heating efficiency. However, in the conventional structure we can see from Fig. 4(b) that a portion of heat flux, which conducts horizontally in $\mathrm{SiO}_{2}$ overlay in the heat deliver process, does not contribute to waveguide heating, resulting in not only lower heating efficiency but also severe thermal crosstalk as discussed below.

Relationship between $d$ and $T_{\text {WGcore }}$ is simulated, as shown in Fig. 5. Since the thermal conductivity of $\mathrm{Si}$ and $\mathrm{SiO}_{2}$ is much higher than that of air, heat flux mainly conducts through BOX and $\mathrm{Si}$ substrate. As the width of graphene nanoheater increases, heat flux generated by the additional-width graphene nanoheater conducts horizontally through graphene and contributes to the waveguide heating, leading to higher $T_{\text {WGcore }}$. It is worth mentioning that although a higher $T_{\text {WGcore }}$ is obtained with a wider graphene nanoheater, it is less powerefficient (as discussed below) since the heating length is reduced for a fixed area of graphene $\left(100 \mu \mathrm{m}^{2}\right.$ in our simulation).

Recent progress introduces air trenches to thermo-optic phase shifters, ${ }^{25,39,40}$ harnessing the poor thermal conductivity of air, further improving the heating efficiency, trading off response speed to some extent though. The structure is schematically shown in Fig. 1(b), where BOX outside graphenenanoheater-covering area is fully etched. Simulated thermal field distribution is shown in Fig. 6(a). $T_{\text {WGcore }}$ is drastically enhanced up to $311.17 \mathrm{~K}$, indicating that the heating efficiency is further improved by a factor of $200 \%$ compared to the structure without air trench. $T_{\mathrm{WGcore}}$ as function of $d$ is shown in Fig. 6(b). As can be seen, $T_{\text {WGcore }}$ decreases with increasing 


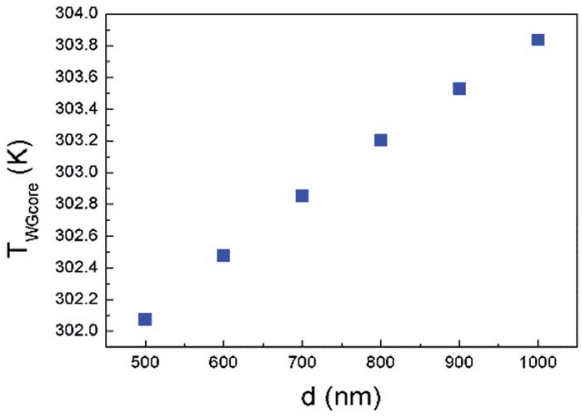

Fig. 5 Relationship between $d$ and waveguide core temperature TWGcore.
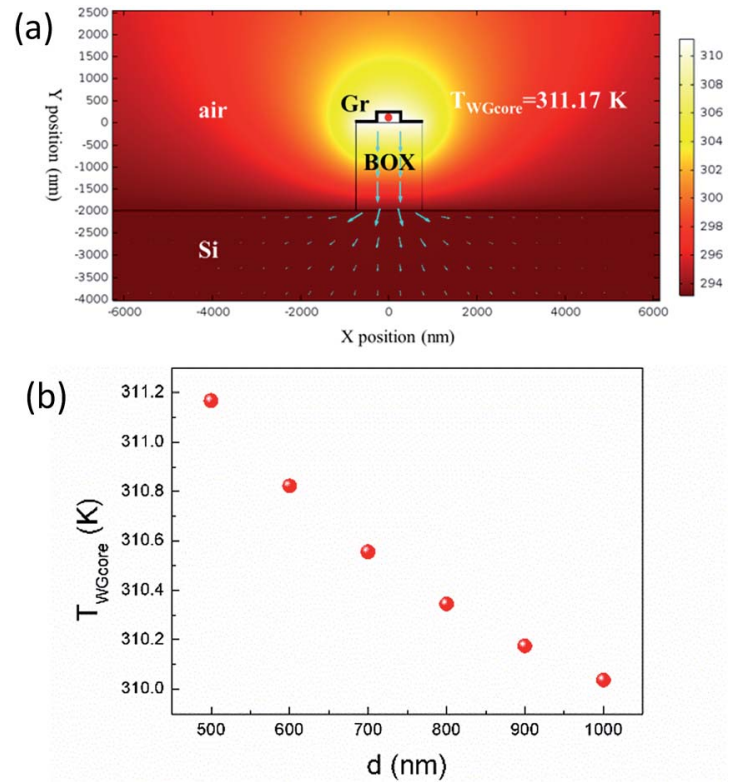

Fig. 6 (a) Thermal field distribution with $1.94 \mu \mathrm{m}$-wide graphene nanoheater. Blue arrows shows directions of heat flux. (b) Waveguide core temperature as function of $d$.

width of graphene nanoheater, showing an obviously opposite trend compared to Fig. 5.

In the structure without air trench, as the width of graphene nanoheater increases, more heat power is generated and then conducts horizontally through graphene and finally contributes to the waveguide heating, resulting in increasing $T_{\text {WGcore }}$. In the structure with air trench, the above-mentioned process is still valid. However, increasing width of graphene nanoheater enables increasing width of heat dissipation path, leading to a decreasing $T_{\mathrm{WG} c o r e}$, countering the effect of additional heating power. As a result, $T_{\mathrm{WGcore}}$ is affected by the more dominating factor, which is the width of heat dissipation path concluded from our simulation.

Power consumption for $\pi$ phase shift $\left(P_{\pi}\right)$ is another important parameter for an OPA's phase shifter, since it is related to the total on-chip power consumption which scales with number of emitters. ${ }^{1}$ Using eqn (1), we calculated the phase shift for the graphene contact-heating structure as

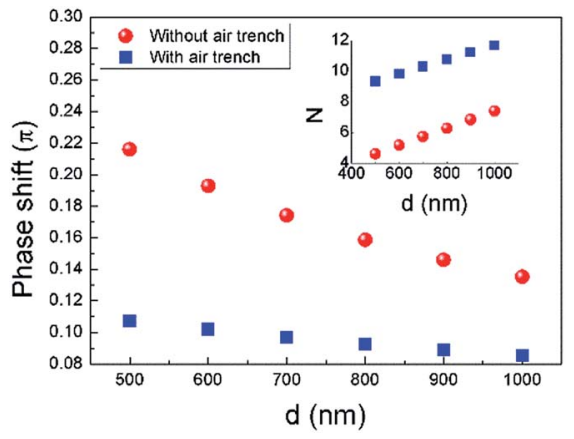

Fig. 7 Phase shift of the proposed structure with and without air trench. Inset shows corresponding $N$ as function of $d$.

function of $d$, as shown in Fig. 7. In order to obtain $\pi$ phase shift, one may operate multiple graphene nanoheaters in series, where number of nanoheaters $N$ equals to reciprocal of phase shift (inset of Fig. 7). However, this method dramatically increases the footprint of the nonradiative phase-shifting region, leading to a lower fill factor. An alternative yet moderately less power-efficient method is operating a single-phase shifter with elevated thermal power. $P_{\pi}$ of the latter case is calculated as shown in Fig. 8(a). A $P_{\pi}$ as low as $4.65 \mathrm{~mW}$ is achieved in graphene contact-heating structure with air trench, approximately half of the state-of-the-art OPAs, as shown in Fig. 8(b).

The transient response is also simulated, as shown in Fig. 9, where $y$-axis is the normalized temperature difference at waveguide core compared to ambient condition ( $\left.\Delta T_{\mathrm{WG}}{ }_{\mathrm{Wcor}}\right)$. The 10$90 \%$ rising time and decaying time for graphene nanoheaters
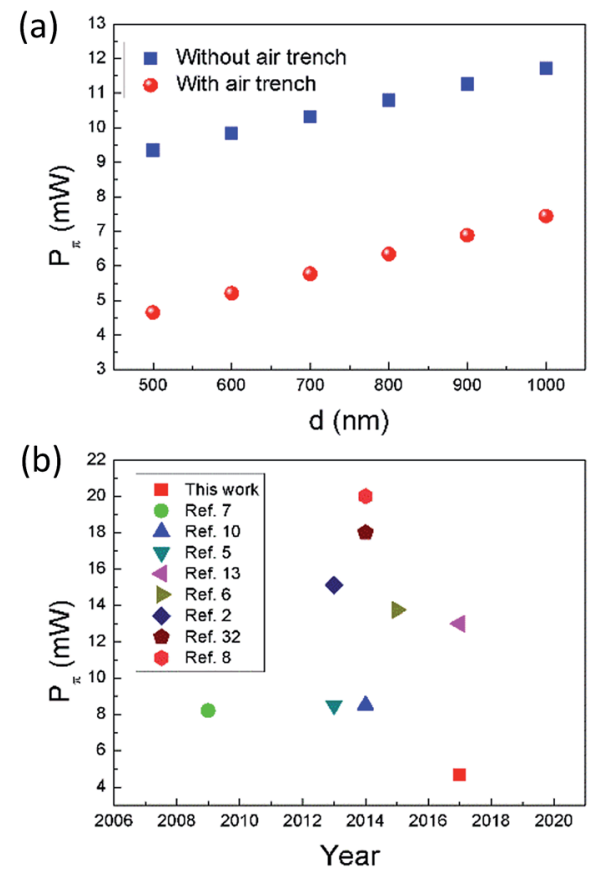

Fig. 8 (a) $P_{\pi}$ as function of $d$ with and without air trench. (b) Summarization of $P_{\pi}$ reported in references. 
(a)

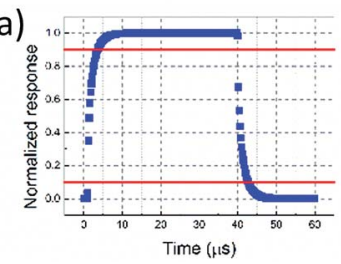

(b)

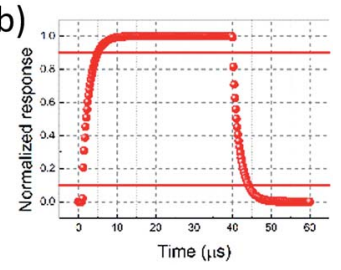

Fig. 9 Transient responses in structure without air trench (a) and with air trench (b).

without air trench are $2.57 \mu$ s and $2.59 \mu$ s. As qualitatively analysed above, the rising time and decaying time for graphene nanoheaters with air trench are relatively longer, which are 3.93 $\mu \mathrm{s}$ and $3.96 \mu \mathrm{s}$, respectively, due to the poor thermal conductivity of air. The response time as function of $d$ in both structures are shown in Fig. 10. The response time in the structure without air trench is proportional to $d$, as opposed to that in the structure with air trench. Explanation to this opposite trend is due to the opposite trend of $T_{\text {WGcore }}$ as function of $d$. Qualitatively speaking, it requires longer time to reach thermal equilibrium for higher $T_{\text {WGcore }}$. The shortest response time is on order of $5.16 \mu \mathrm{s}$, corresponding to an operation speed on order of $190 \mathrm{kHz}$, which is, to our best knowledge, the fastest thermally tuned OPA. Please note this operation speed is limited by $10-90 \%$ response time, unlike ref. 41 in which the operation speed is determined by half-maximum steering angle. Following ref. 41, we would achieve a corresponding operation speed on order of $200 \mathrm{kHz}$.

Thermal crosstalk is detrimental for OPAs, especially for the large-scale integrated ones, where each emitter requires an

(a)

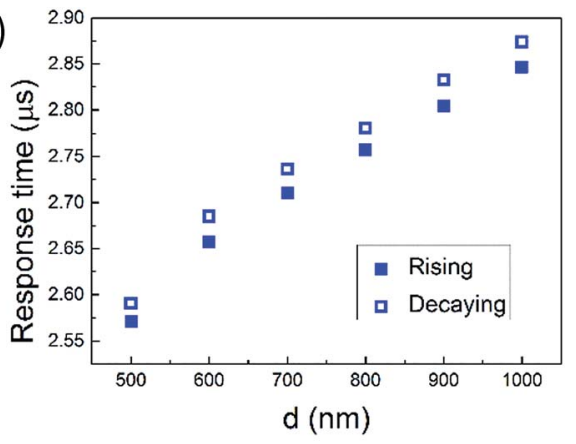

(b)

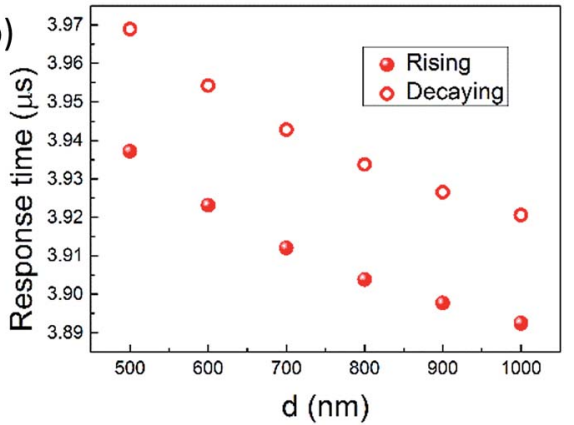

Fig. 10 Rising and decaying time as function of $d$ in the structure without air trench (a) and with air trench (b). independent thermo-optic phase shifter in order to balance phase difference resulted from fabrication imperfection. ${ }^{11,16}$ Therefore low thermal crosstalk is another design criterion. Thermal crosstalk among emitters is simulated by applying 1 $\mathrm{mW}$ heating power to all graphene nanoheaters except for the central one,$^{16}$ as shown in Fig. 11. A higher temperature at the central waveguide means severer thermal crosstalk. The width of graphene nanoheaters are $1.94 \mu \mathrm{m}(d=500 \mathrm{~nm})$ and distances between adjacent waveguides are $4 \mu \mathrm{m}\left(d_{\mathrm{WG}}=4 \mu \mathrm{m}\right)$.

As shown in Fig. 11(a)-(c), the waveguide core temperature in the conventional structure and the proposed graphene contactheating structures without and with air trenches are $295.75 \mathrm{~K}$, $294.27 \mathrm{~K}$ and $293.73 \mathrm{~K}$, respectively, corresponding to temperature elevations of $2.6 \mathrm{~K}, 1.1 \mathrm{~K}$ and $0.58 \mathrm{~K}$ at the central waveguides, respectively, indicating ameliorated thermal crosstalk between channels in the graphene contact-heating structures. Thermal crosstalk in the graphene contact-heating structure with air trench is reduced by a factor of $448 \%$ compared to the conventional structure. In the conventional structure, heat flux conducts vertically through $\mathrm{SiO}_{2}$, heating up the waveguide underneath, as well as horizontally to adjacent waveguides in the heat deliver process, leading to severe thermal crosstalk. However, in the graphene contact-heating structure, heating flux generated by graphene flows directly to the Si waveguide. Furthermore, the presence of air trenches introduces heat
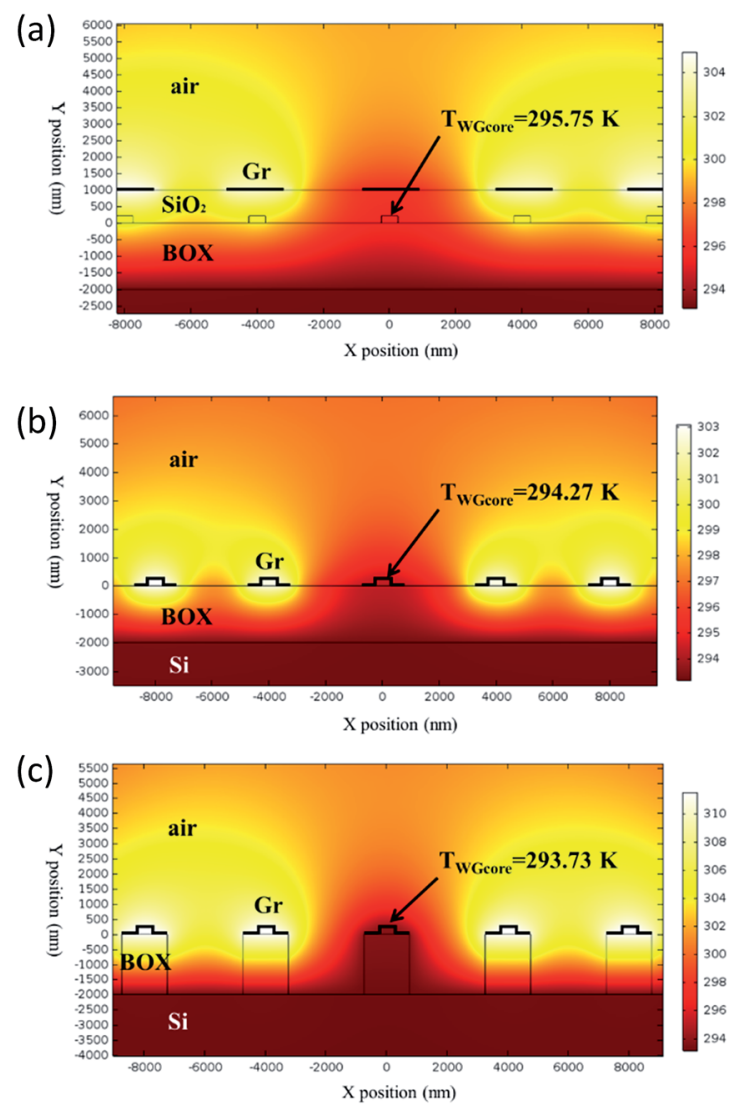

Fig. 11 Thermal field distributions of conventional structure with 1 $\mu \mathrm{m}$-thick $\mathrm{SiO}_{2}$ top cladding (a), graphene contact-heating structure without air trench (b) and with air trench (c). 
isolation among channels that significantly diminishes thermal crosstalk. Dependence of the temperature at central waveguide core $\left(T_{\text {WGcore central }}\right)$ on waveguide pitch $\left(d_{\mathrm{WG}}\right)$ with and without air trench is shown in Fig. 12. It can be seen that when $d_{\mathrm{WG}}$ is smaller than $4 \mu \mathrm{m}$, the central waveguide suffers from severe thermal crosstalk in both structures, although thermal crosstalk in the structure with air trench is approximately half of that in the structure without air trench. However, when $d_{\mathrm{WG}}$ is larger than $10 \mu \mathrm{m}$, thermal crosstalk is negligible. It is worth mentioning that the waveguide pitch in the conventional structure is generally on order of $100 \mu \mathrm{m}$ to reduce thermal crosstalks. ${ }^{16}$ Using graphene nanoheaters, the waveguide pitch can be reduced by one order of magnitude, giving rise to significantly reduced size of OPA chip, footprint of nonradiative phase-shifting area and consequently enlarged fill factor.

\section{Beam steering in $\theta$ direction}

Achieving wide steering range in the $\phi$ direction requires smaller antenna pitch and is only an issue of nano-processing technology. However, beam steering in the $\theta$ direction is much more challenging. Generally speaking, in an ideal OPA, beam steering range in $\theta$ direction should be as wide as possible. However, for some realistic applications, such as collision avoidance for autonomous driving at $100 \mathrm{~m}$ distance or longer, beam steering as wide as 2 degree is practically enough since the angle subtended from a pedestrian or a vehicle is merely on order of 1 degree.

In a grating emitter, each grating tooth can be regarded as an antenna. Therefore, by operating a graphene nanoheater parallel to the $y$-axis at the beginning of the antenna array (Fig. 1(a)), a temperature gradient is generated parallel to the $x$ axis, resulting in beam steering in the $\theta$ direction.

The grating pitch and duty cycle modeled in this paper are $600 \mathrm{~nm}$ and $50 \%$, respectively. Using $2 \mathrm{D}$ symmetry, the polar plot of the upward-radiated far-field pattern is shown in Fig. 13(a), with peak radiation direction at $78.8^{\circ}$ and a beam width of $5^{\circ}$ (full width at half maximum). Beam steering at various heating power is shown in Fig. 13(b). For $30 \mathrm{~mW}$ the peak radiation direction is at $76.5^{\circ}$, indicating a beam steering of $2.3^{\circ}$ is achieved. The electric field and thermal field distribution corresponding to $30 \mathrm{~mW}$ are shown in Fig. 13(c).

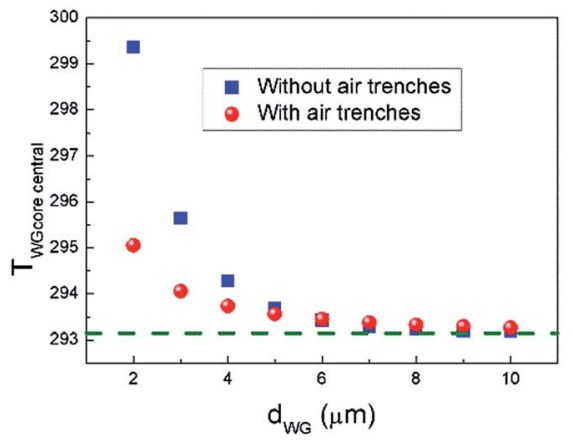

Fig. 12 Dependence of temperature at central waveguide core ( $\left.T_{\text {WGcore central }}\right)$ on waveguide pitch $\left(d_{\text {WG }}\right)$ with and without air trench. Dashed green line indicates the ambient temperature.
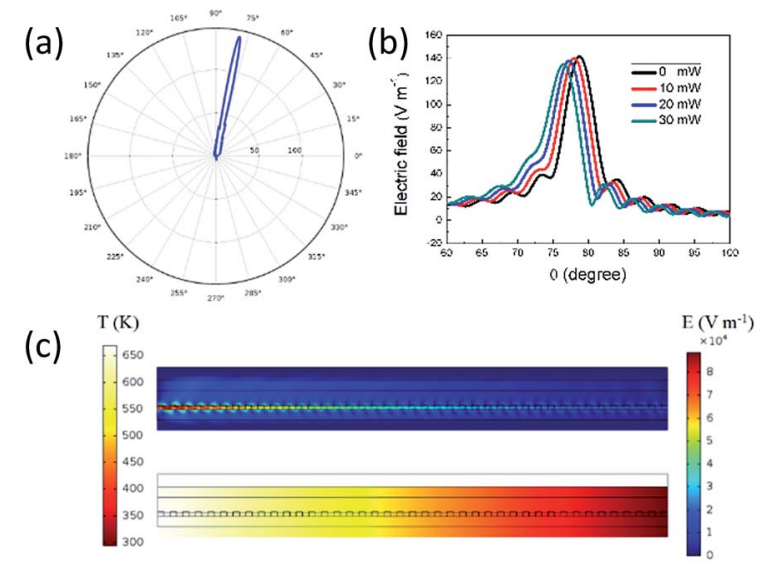

Fig. 13 (a) Polar plot of the far-field radiation pattern. (b) Beam steering for $0-30 \mathrm{~mW}$ heating power with $10 \mathrm{~mW}$ step. (c) Electric field distribution (upper panel) and thermal field distribution (lower panel) corresponding to $30 \mathrm{~mW}$.

\section{Conclusion}

In this paper, improved performances of OPAs assisted by transparent graphene nanoheaters have been demonstrated. First, assisted by transparent graphene nanoheaters, the heating efficiency is enhanced by $62.96 \%$ compared to the conventional structure with $1 \mu \mathrm{m} \mathrm{SiO} \mathrm{Si}_{2}$ overlay, and is further enhanced by a factor of $200 \%$ by the presence of air trench. In addition, the operation speed is on order of $200 \mathrm{kHz}$, twice the previously reported fastest OPA. Moreover, power consumption for $\pi$ phase shift is as low as $4.65 \mathrm{~mW}$, approximately half of the state-of-theart OPAs. Furthermore, thanks to the poor thermal conductivity of air, thermal crosstalk is reduced by factor of $448 \%$ compared to the conventional structure, leading to footprint of nonradiative area reduced by one order of magnitude and consequently significantly enlarged fill factor. Finally, by applying $30 \mathrm{~mW}$ heating power, beam steering of $2.3^{\circ}$ is achieved, satisfying applications such as collision avoidance for autonomous driving.

\section{Conflicts of interest}

There are no conflicts to declare.

\section{Acknowledgements}

This work was supported by the National Key Research and Development Project [2017YFB0405102], the National Natural Science Foundation of China (NSFC) [61727822, 61674148, 11674314, 11774343], the Frontier Science Research Project of Chinese Academy of Sciences [QYZDY-SSW-JSC006] and the Science and Technology Development Plan of Jilin Province [20170204013GX].

\section{Notes and references}

1 M. J. R. Heck, Highly integrated optical phased arrays: photonic integrated circuits for optical beam shaping and beam steering, Nanophotonics, 2016, 6(1), 93-107. 
2 T. Cao, G. Zheng and S. Wang, Chemical control of continuous light-steering using an array of gradient $\mathrm{Au} /$ Bi2Se3/Au strips, RSC Adv., 2015, 5(85), 69319-69324.

3 T. Cao, Y. Zou, A. M. Adawi and M. J. Cryan, Directive emission of red conjugated polymer embedded within zero index metamaterials, Opt. Express, 2014, 22(19), 2269922706.

4 T. Cao, G. Zheng, S. Wang and C. Wei, Ultrafast beam steering using gradient $\mathrm{Au}-\mathrm{Ge}_{2} \mathrm{Sb}_{2} \mathrm{Te}_{5}-\mathrm{Au}$ plasmonic resonators, Opt. Express, 2015, 23(14), 18029.

5 T. Cao, C. Wei, R. E. Simpson, L. Zhang and M. J. Cryan, Broadband Polarization-Independent Perfect Absorber Using a Phase-Change Metamaterial at Visible Frequencies, Sci. Rep., 2014, 4(2), 3955.

6 T. Cao, C. Wei, R. E. Simpson, L. Zhang and M. J. Cryan, Rapid phase transition of a phase-change metamaterial perfect absorber, Opt. Mater. Express, 2013, 3(8), 1101-1110.

7 H. Nikkhah, K. V. Acoleyen and R. Baets, Beam steering for wireless optical links based on an optical phased array in silicon, Ann. Telecommun, 2013, 68(1-2), 57-62.

8 C. V. Poulton, A. Yaacobi, D. B. Cole, M. J. Byrd, M. Raval, D. Vermeulen and M. R. Watts, Coherent solid-state LIDAR with silicon photonic optical phased arrays, Opt. Lett., 2017, 42(20), 4091.

9 D. N. Hutchison, J. Sun, J. K. Doylend, R. Kumar, J. Heck, W. Kim, C. T. Phare, A. Feshali and H. Rong, Highresolution aliasing-free optical beam steering, Optica, 2016, $3(8), 887$.

10 J. Sun, E. Timurdogan, A. Yaacobi, E. S. Hosseini and M. R. Watts, Large-scale nanophotonic phased array, Nature, 2013, 493(7431), 195.

$11 \mathrm{H}$. Abediasl and H. Hashemi, Monolithic optical phasedarray transceiver in a standard SOI CMOS process, Opt. Express, 2015, 23(5), 6509.

12 K. V. Acoleyen, W. Bogaerts, J. Jágerská, N. L. Thomas, R. Houdre and R. Beats, Off-chip beam steering with a one-dimensional optical phased array on silicon-oninsulator, Opt. Lett., 2009, 34(9), 1477.

13 D. Kwong, A. Hosseini, J. Covey, Y. Zhang, X. Xu, H. Subbaraman and R. T. Chen, On-chip silicon optical phased array for two-dimensional beam steering, Opt. Lett., 2014, 39(4), 941.

14 K. V. Acoleyen, H. Rogier and R. Beats, Two-dimensional optical phased array antenna on silicon-on-insulator, Opt. Express, 2010, 18(13), 13655.

15 J. Sun, E. S. Hosseini, A. Yaacobi, D. B. Cole, G. Leake, D. Coolbaaugh and M. R. Watts, Two-dimensional apodized silicon photonic phased arrays, Opt. Lett., 2014, 39(2), 367.

16 J. K. Doylend, M. J. R. Heck, J. T. Bovington, J. D. Peters, L. A. Coldren and J. E. Bowers, Two-dimensional free-space beam steering with an optical phased array on silicon-oninsulator, Opt. Express, 2011, 19(22), 21595.

17 T. Komljenovic, R. Helkey, L. Coldren and J. E. Bowers, Sparse aperiodic arrays for optical beam forming and LIDAR, Opt. Express, 2017, 25(3), 2511.
18 L. Tong, F. Qiu, T. Zeng, J. Long, J. Yang, R. Wang, J. Zhang, C. Wang, T. Sun and Y. Yang, Recent progress in the preparation and application of quantum dots/graphene composite materials, RSC Adv., 2017, 7(76), 47999-48018.

19 Z. Zhu, I. Murtaza, H. Meng and W. Huang, Thin film transistors based on two dimensional graphene and graphene/semiconductor heterojunctions, RSC Adv., 2017, 7(28), 17387-17397.

$20 \mathrm{~J}$. Li, L. Niu, Z. Zheng and F. Yan, Photosensitive Graphene Transistors, Adv. Mater., 2014, 26(31), 5239.

21 R. R. Nair, P. Blake, A. N. Grigorenko, K. S. Novoselov, T. J. Booth, T. Stauber, N. M. R. Peres and A. K. Geim, Fine Structure Constant Defines Visual Transparency of Graphene, Science, 2008, 320(5881), 1308.

22 L. Yu, Y. Yin, Y. Shi, D. Dai and S. He, Thermally tunable silicon photonic microdisk resonator with transparent graphene nanoheaters, Optica, 2016, 3(2), 159.

23 A. A. Balandin, S. Ghosh, W. Bao, I. Calizo, D. Teweldebrhan, F. Miao and C. N. Lau, Superior thermal conductivity of single-layer graphene, Nano Lett., 2008, 8(3), 902.

24 S. Gan, C. Cheng, Y. Zhan, B. Huang, X. Gan, S. Li, S. Lin, X. Li, J. Zhao, H. Chen and Q. Bao, A highly efficient thermo-optic microring modulator assisted by graphene, Nanoscale, 2015, 7(47), 20249-20255.

25 Y. Sun, Y. Cao, Y. Yi, L. Tian, Y. Zheng, J. Zheng, F. Wang and D. Zhang, A low-power consumption MZI thermal optical switch with a graphene-assisted heating layer and air trench,, RSC Adv., 2017, 7(63), 39922-39927.

26 A. Pospischil, M. Humer, M. M. Furchi, D. Bachmann, R. Guider, T. Fromherz and T. Mueller, CMOS-compatible graphene photodetector covering all optical communication bands, Nat. Photonics, 2013, 7(11), 892-896.

27 Y. Han, L. Zhang, X. Zhang, K. Ruan, L. Cui, Y. Wang, L. Liao, Z. Wang and J. Jie, Clean surface transfer of graphene films via an effective sandwich method for organic light-emitting diode applications, J. Mater. Chem. C, 2013, 2(1), 201-207.

28 X. Liang, B. A. Sperling, I. Calizo, G. Cheng, C. A. Hacker, Q. Zhang, Y. Obeng, K. Yan, H. Peng, Q. Li, X. Zhu, H. Yuan, A. R. H. Walker, Z. Liu, L. Peng and C. A. Richter, Toward clean and crackless transfer of graphene, ACS Nano, 2011, 5(11), 9144.

29 Y. Lee, S. Bae, H. Jang, S. Jang, S. Zhu, S. H. Sim, Y. I. Song, B. H. Hong and J. Ahn, Wafer-Scale Synthesis and Transfer of Graphene Films, Nano Lett., 2010, 10(2), 490.

30 H. Li, Y. Anugrah, S. J. Koester and M. Li, Optical absorption in graphene integrated on silicon waveguides, Appl. Phys. Lett., 2012, 101(11), 611-622.

31 N. Youngblood, Y. Anugrah, R. Ma, S. J. Koester and M. Li, Multifunctional graphene optical modulator and photodetector integrated on silicon waveguides, Nano Lett., 2014, 14(5), 2741.

32 M. Liu, X. Yin, E. Ulin-Avila, B. Geng, T. Zentgraf, L. Ju, F. Wang and $\mathrm{X}$. Zhang, A graphene-based broadband optical modulator, Nature, 2011, 474(7349), 64.

33 M. Liu, X. Yin and X. Zhang, Double-Layer Graphene Optical Modulator, Nano Lett., 2012, 12(3), 1482. 
34 W. Li, B. Chen, C. Meng, W. Fang, Y. Xiao, X. Li, Z. Hu, Y. Xu, L. Tong, H. Wang, W. Liu, J. Bao and Y. R. Shen, Ultrafast AllOptical Graphene Modulator, Nano Lett., 2014, 14(2), 955.

35 M. C. Lemme, F. H. L. Koppens, A. L. Falk, M. S. Rudner, H. Park, L. S. Levitov and C. M. Marcus, Gate-activated photoresponse in a graphene $\mathrm{p}-\mathrm{n}$ junction, Nano Lett., 2011, 11(10), 4134.

36 X. Li, D. Xie, H. Park, M. Zhu, T. H. Zeng, K. Wang, J. Wei, $\mathrm{D}$. $\mathrm{Wu}, \mathrm{J}$. Kong and $\mathrm{H}$. Zhu, Ion doping of graphene for high-efficiency heterojunction solar cells, Nanoscale, 2013, 5(5), 1945-1948.

37 E. C. Peters, E. J. H. Lee, M. Burghard and K. Kern, Gate dependent photocurrents at a graphene p-n junction, Appl. Phys. Lett., 2010, 97(19), 193102.

38 C. O. Kim, S. Kim, D. H. Shin, S. S. Kang, J. M. Kim, C. W. Jang, S. S. Joo, J. S. Lee, J. H. Kim, S. Choi and
E. Hwang, High photoresponsivity in an all-graphene $\mathrm{p}-\mathrm{n}$ vertical junction photodetector, Nat. Commun., 2014, 5(2), 3249.

39 Y. F. Liu, X. B. Wang, J. W. Sun, J. Sun, F. Wang, C. M. Chen, X. Q. Sun, Z. C. Cui and D. M. Zhang, Improved performance of thermal-optic switch using polymer/silica hybrid and air trench waveguide structures, Opt. Lett., 2015, 40(9), 18881891.

40 Y. F. Liu, X. B. Wang, J. Sun, H. J. Gu, X. Q. Sun, C. M. Chen, F. Wang and D. M. Zhang, Thermal field analysis of polymer/ silica hybrid waveguide thermo-optic switch, Opt. Commun., 2015, 356, 79-83.

41 A. Yaacobi, J. Sun, M. Moresco, G. Leake, D. Coolbaugh and M. R. Watts, Integrated phased array for wide-angle beam steering, Opt. Lett., 2014, 39(15), 4575. 